\title{
Entrapment of motor nerves in motor neuron disease: does double crush occur?
}

\author{
Vinay Chaudhry, Lora L Clawson
}

\begin{abstract}
Objective-To investigate whether "diseased nerves" are more prone to entrapment neuropathy than normal nerves. Nerve conduction studies of human neuropathies have shown that electrophysiological abnormalities are often most prominent at potential sites of nerve entrapment, and entrapments are more common in patients with radiculopathies - a concept designated as "double crush". As entrapment neuropathies commonly occur in otherwise healthy subjects, it is unclear whether this relation is coincidental or whether peripheral nerves affected by disease are rendered more susceptible to effects of repeated minor trauma, traction, or mechanical compression.
\end{abstract}

Methods-Sequential ulnar nerve conduction studies were prospectively performed at baseline and at four, eight, and 12 month intervals in 16 patients with amyotrophic lateral sclerosis. Ulnar nerve entrapment was defined as a focal reduction $(>10 \mathrm{~m} / \mathrm{s})$ in conduction velocity in the across-elbow segment.

Results-Ulnar sensory and motor nerve fibres showed similar findings of ulnar nerve entrapment at baseline and at follow up over the period of the study. Nerves with ulnar nerve entrapment showed a significantly greater reduction in distal motor amplitudes than nerves without entrapment, even though distal ulnar sensory amplitudes remained unchanged.

Conclusions-Motor nerves in motor neuron disease do not seem to be more susceptible to entrapment at the elbow than do healthy sensory nerves, thus casting doubt on the double crush hypothesis. Nerves with double pathology (amyotrophic lateral sclerosis and ulnar nerve entrapment), however, seem to undergo more rapid axonal loss than do nerves with single pathology (amyotrophic lateral sclerosis or ulnar nerve entrapment alone).

$(\mathcal{F}$ Neurol Neurosurg Psychiatry 1997;62:71-76)

Neurology, John

Hopkins University

School of Medicine,

Baltimore, MD, USA

V Chaudhry

L L Clawson

Correspondence to:

DrV Chaudhry, Department

of Neurology, Johns Hopkins

Hospital, Pathology 509,

Baltimore, MD 21287, USA.

Received 11 March 1996

and in revised form

22 May 1996

Accepted 16 August 1996
This concept has been used to explain the clinical finding that patients with proximal lesions such as cervical radiculopathies or thoracic outlet syndrome are more likely to have distal lesions such as median nerve entrapment at the wrist (carpal tunnel syndrome) or ulnar nerve entrapment at the elbow. ${ }^{2-9}$ Experimentally, Shimpo et $a l^{10}$ showed that guinea pigs that had a constricting ligature placed proximally in the sciatic nerve developed plantar neuropathy in the ipsilateral foot but not on the contralateral foot. Peripheral nerves also seem to be more vulnerable to compressive injury in the presence of generalised neuropathies such as diabetes or Guillain-Barré syndrome. ${ }^{11}{ }^{12}$ Hopkins and Morgan-Hughes ${ }^{13}$ were able to show that guinea pigs with demyelinating neuropathy induced by diphtheria toxin developed compressive neuropathies in their footpads sooner than did healthy animals.

As well as the increased incidence of a second lesion in the presence of an already existing lesion, double crush syndrome has also been interpreted to mean that two sequential lesions can be additive. ${ }^{14-16}$ Therefore, the presence of one proximal lesion will lessen the nerve's ability to withstand additional distal compression. Hence, trauma, traction, or mechanical compression at a potential site of entrapment may not be sufficient to cause clinically important symptoms in healthy subjects, but may do so if that nerve is already affected by another disease process.

Although double crush is an attractive concept, little clinical data support it. In this study we tested the double crush hypothesis by prospectively studying ulnar nerves in patients with amyotrophic lateral sclerosis. Amyotrophic lateral sclerosis provides a model in which motor fibres are "diseased" and sensory fibres are healthy within the same nerve fibre bundle, thus allowing comparison of diseased and normal fibres at the same site. Firstly, we reasoned that if the concept of double crush is correct, ulnar motor fibres in patients with amyotrophic lateral sclerosis will show a higher incidence of ulnar neuropathy at the elbow than would the adjacent ulnar sensory fibres, which do not have a superimposed pathology but are exposed to the same external factors that lead to ulnar nerve entrapment. Secondly, if the effect of two lesions is additive, then ulnar motor nerves that develop ulnar nerve entrapment will show a greater axonal loss than ulnar motor nerves that do not have ulnar nerve entrapment. Lastly, ulnar sensory fibres that have only one lesion will show minimal axonal loss compared with ulnar motor fibres that have two lesions (amy- 
Figure 1 Comparison of baseline (filled squares) and follow up (open squares) distal evoked amplitudes in ulnar to ADM (abductor digiti minimi) and ulnar to $F D$ (first dorsal interosseous) muscles on the left $(L)$ and right $(R)$ sides. There is a significant reduction in CMAP amplitude $(P$ values represent comparison of baseline to follow up by paired $t$ test).
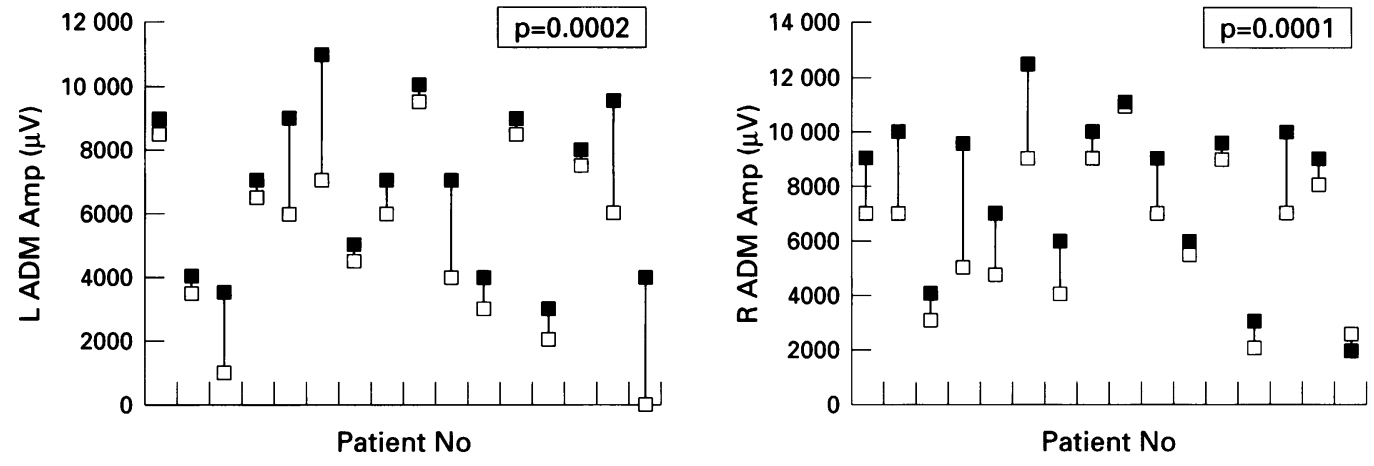

Baseline

Follow up

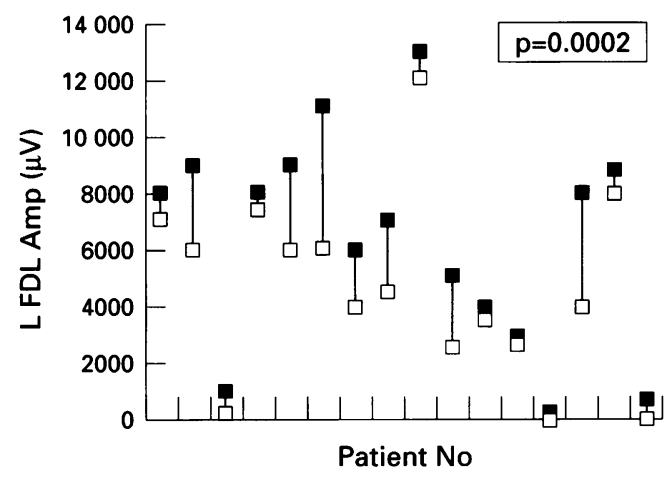

otrophic lateral sclerosis and ulnar nerve entrapment).

\section{Patients and methods}

Serial nerve conduction studies were performed at baseline, and at four, eight, and 12 month intervals in 16 patients (nine men and seven women) with amyotrophic lateral sclerosis. None of the patients had sensory symptoms or clinical evidence of selective ulnar neuropathy at the elbow. The average age was 54 (range 33 to 75 ) years. In each case, bilateral ulnar nerve conduction studies were performed. The stimulation sites were the wrist, elbow, above-elbow, and axilla, with the elbow maintained at $135^{\circ}$ flexion. The recording sites for the ulnar motor nerve conduction studies were the abductor digiti minimi (ADM) and the first dorsal interosseous (FDI) muscles. The recording site for the ulnar sensory nerve conduction studies was the fifth digit. The stimulation and recording sites were all precisely predefined: the stimulation site at the wrist was $65 \mathrm{~mm}$ from the recording site; below-elbow and above-elbow segments were each $5 \mathrm{~cm}$ away from the olecranon process; and the axilla segment was $10 \mathrm{~cm}$ proximal to the above-elbow site. The active recording sites for the ADM and FDI were the midpoint of the hypothenar eminence and the first metacarpal bone dorsally, respectively; the corresponding reference electrode was placed on the metacarpophalangeal joints of the fifth and the second digits. The recording sites for the sensory fibres were the distal and proximal creases of the fifth digit. Skin temperature was recorded in the second digit, and the limb heated up when required to bring the recorded temperature to $>32^{\circ} \mathrm{C}$. The same examiner

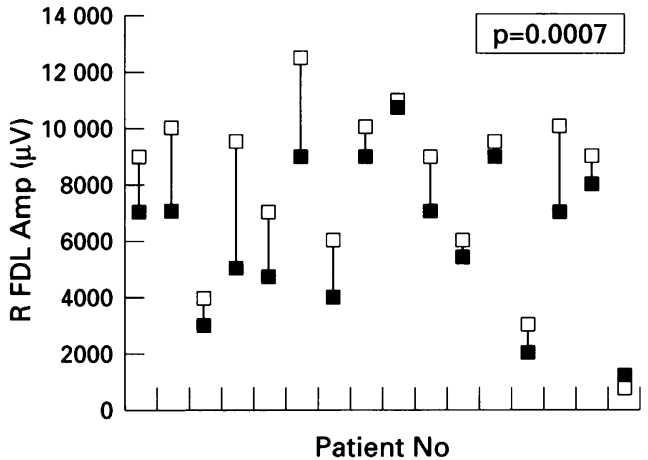

(VC) performed the conductions in each patient. Supramaximal stimulations were ensured and five responses were averaged for the sensory recordings. Sensory nerve action potential (SNAP) and compound muscle action potential (CMAP) amplitudes (peak to peak for SNAP; baseline to peak for CMAP) and conduction velocities were recorded for each segment. Electrophysiologically, ulnar nerve entrapment was said to be present if the conduction velocity in the across-the-elbow segment was less than that in the below-elbow forearm segment by $10 \mathrm{~m} / \mathrm{s}$ or more. Data were analysed separately for conduction velocity reduction in sensory fibres, motor fibres, and both sensory and motor fibres. The baseline and follow up measures were analysed by paired $t$ test. The institutional review board approved the protocol and informed consent was obtained for each patient.

\section{Results}

Thirty two ulnar sensory and 64 ulnar motor nerve conduction studies were performed at baseline. All patients had nerve conduction studies at baseline and at least one follow up study, with the median time from baseline to follow up being eight (range, 4-12) months. Figure 1 shows baseline and final follow up measurements of CMAP amplitudes for bilateral ADM and FDI muscles. In most patients, CMAP amplitudes fell between the time of baseline and follow up examinations, with the average reductions being $25 \%$ for the left $\mathrm{ADM}$ muscle, $20 \%$ for the right $\mathrm{ADM}$ muscle, $35 \%$ for the left FDI muscle, and $34 \%$ for the left FDI muscle. By contrast, ulnar sensory fibres (fig 2) showed no significant change in ampli- 
Figure 2 Comparison of baseline (filled squares) squares) distal evoked sensory amplitudes in ulnar to digit 5 on the left $(L)$ and right $(R)$ sides. Most patients show no significant change. and follow up (open
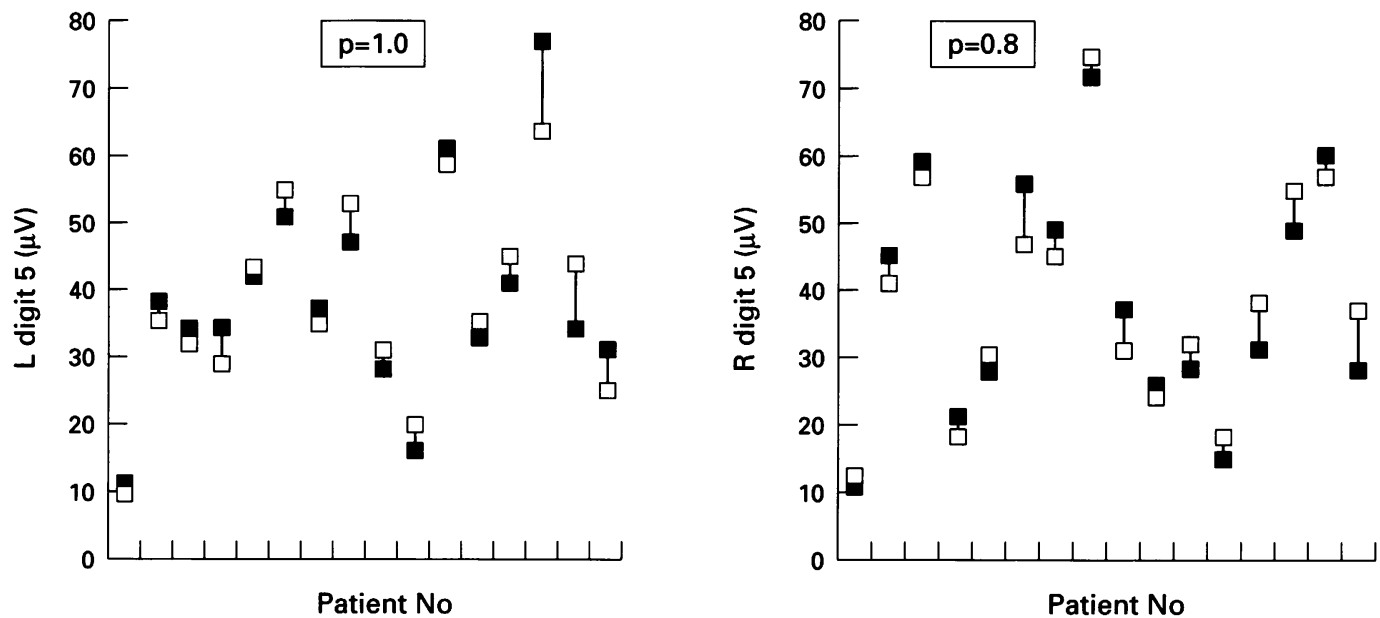

Figure 3 Incidences of focal reductions of $\geqslant 10$ $\mathrm{m} / \mathrm{s}$ in conduction velocities in the across-elbow segment in only sensory, only motor, and in both sensory and motor nerve fibres, at baseline and at follow up. There was no significant difference from baseline to follow up in the percentage of nerves showing the finding of ulnar nerve entrapment.
Figure 4 The focal change in conduction velocity in the across-elbow segment (below-elbow forearm segment - acrosselbow segment) is plotted against the CMAP amplitude for all recording sites and for all baseline and follow up evaluations. Ulnar nerve entrapment is defined by a focal reduction in this segment of $>10 \mathrm{~m} / \mathrm{s}$ (to the right of the vertical dotted line). The horizontal dotted line represents the lower limit of normal for the CMAP amplitude in our laboratory.
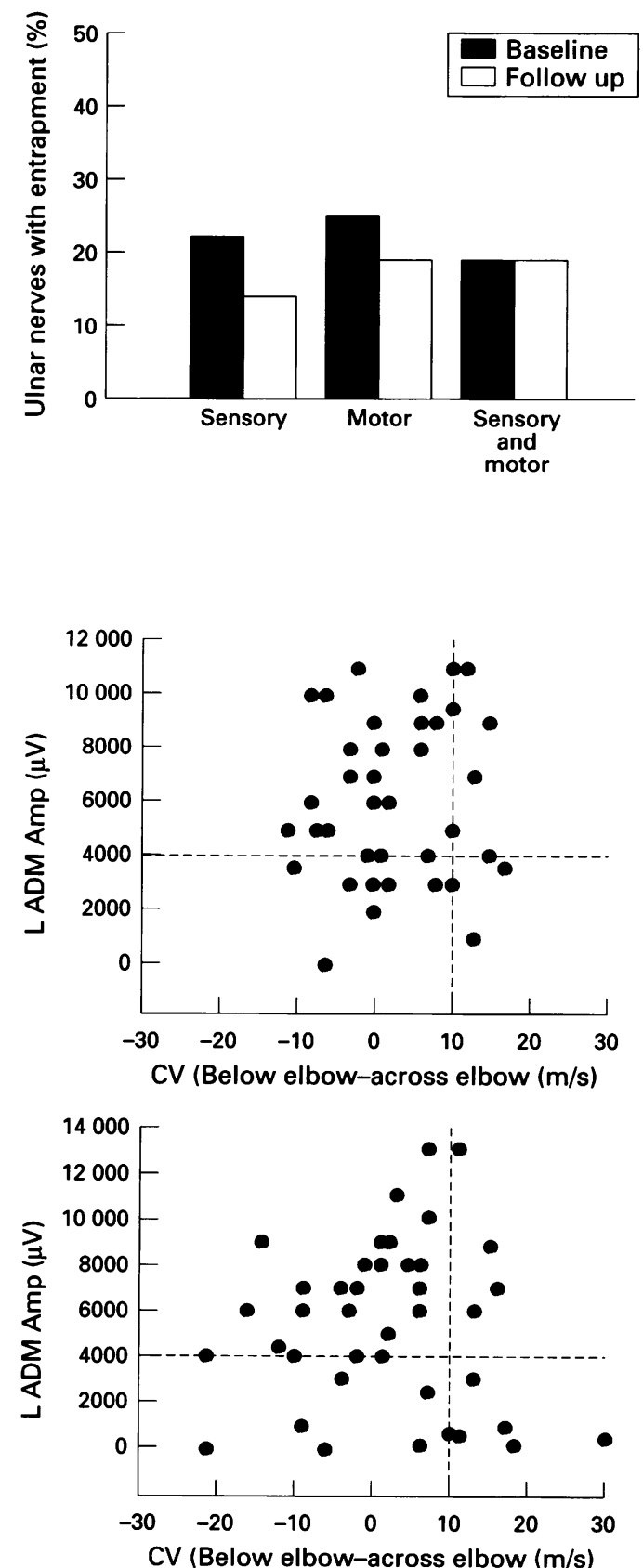

tude, with the average reduction being $2 \%$. These findings established that ulnar motor fibres innervating all four muscles were indeed "diseased" and undergoing Wallerian-like degeneration, as shown by progressive reduction in CMAP amplitudes, whereas the ulnar sensory fibres were relatively "healthy" over the study period. No patient showed evidence of distal ulnar neuropathy; distal motor latencies and sensory conduction velocities in the wrist and second digit segments, were normal in all patients.

At baseline evaluation, ulnar nerve entrapment was found in $22 \%$ of sensory nerves, $25 \%$ of motor nerves, and $19 \%$ in both sensory and motor nerves; on follow up evaluation, these numbers were $14 \%$ for ulnar sensory nerve entrapment, $19 \%$ for ulnar motor nerve entrapment, and $19 \%$ for both. These differences
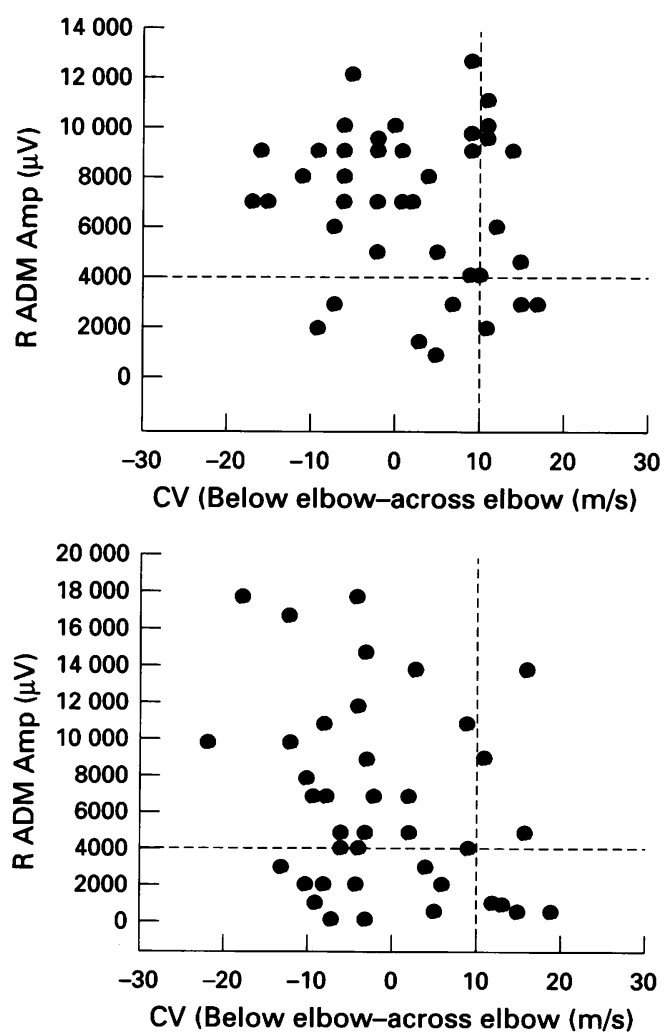
Figure 5 Reduction in evoked amplitudes in abductor digiti minimi $(A D M)$, first dorsal interosseous (FDI), and sensory (digit 2) nerves with entrapment and in nerves without entrapment. A significantly greater reduction in motor amplitudes (FDI and $A D M$ ) was found in nerves with a finding of entrapment.

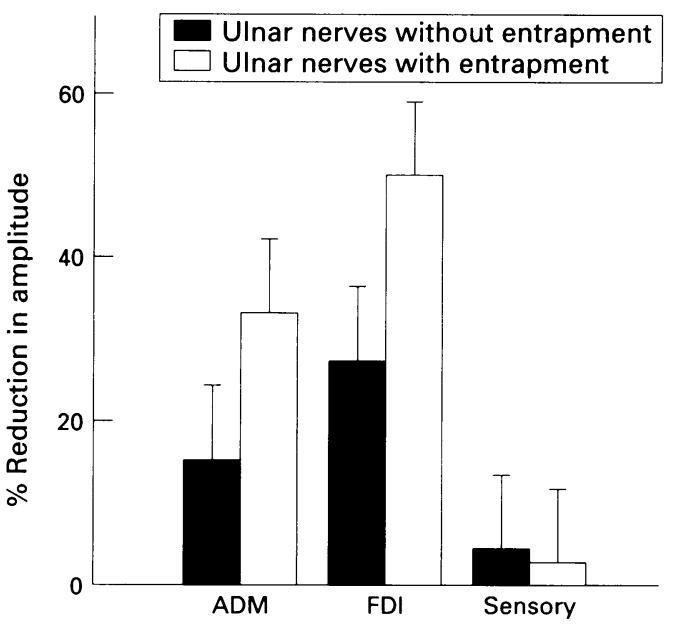

were not significant (fig 3), indicating that frequency of ulnar nerve entrapment does not increase with progressive reduction in CMAP amplitudes. These data were further analysed to determine whether CMAP amplitudes correlated with change in conduction velocity in the across-elbow segment. Differences in conduction velocity between the forearm segment and the across-elbow segment were plotted against the CMAP amplitudes, at baseline and all follow up evaluations, for all four muscles (fig 4). Change in conduction velocity in the acrosselbow segment did not correlate with CMAP amplitudes, again confirming that loss of amplitude did not result in ulnar nerve entrapment.

To test the second part of the hypothesisthat two sequential lesions can add to each other's effects-we compared the percentage reductions in amplitude for sensory and motor fibres in the entrapped ulnar nerves with those of the non-entrapped ulnar nerves (fig 5). As reduction in sensory conduction velocity in the across-elbow segment is considered the most sensitive indicator of ulnar nerve entrapment, ${ }^{17}$ we used ulnar sensory nerve entrapment to analyse these data, although analysis using ulnar motor nerve entrapment gave similar results. For both ADM and FDI recordings, the nerves with entrapment showed a greater reduction in CMAP amplitudes than did nonentrapped ulnar nerves. By contrast, the ulnar sensory fibres with ulnar nerve entrapment did not show a difference in SNAP amplitudes compared with those ulnar sensory fibres not showing a finding of ulnar nerve entrapment. In patients who had unilateral entrapment shown by electrophysiology, we compared side to side differences in amplitude (fig 6). In the same patients, the sides showing the ulnar nerve entrapment had significantly greater reductions in CMAP amplitudes than the sides without ulnar nerve entrapment. Again, the sensory fibres did not show comparable differences in SNAP amplitudes between the two sides. Figure 7 illustrates this point for one patient in whom this difference was most prominent. The left ulnar nerve showed a $31 \%$ reduction in conduction velocity across the elbow segment at the eight month study. In the right ulnar nerve no reduction in conduction velocity was detected in the across-elbow segment. Between the eighth and 12th month periods, a precipitous reduction (99\%) in CMAP amplitude was detected on the left side. At baseline the left ulnar nerve had a relatively normal CMAP amplitude compared with that of the right ulnar nerve, which had already undergone axonal degeneration. Side to side differences in SNAP amplitudes in this patient were minimal.

\section{Discussion}

The initial hypothesis of double crush syndrome was based on the increased incidence of carpal tunnel syndrome or ulnar nerve entrapment in patients with cervical radiculopathy. ${ }^{2}$ Although nerve entrapment seemed well documented electrophysiologically, the presence or distribution of radiculopathy was not. Other
Figure 6 Side to side comparison of reduction in sensory nerve action potential (SNAP) and compound muscle action potential (CMAP) amplitudes in nerves with and without entrapment.
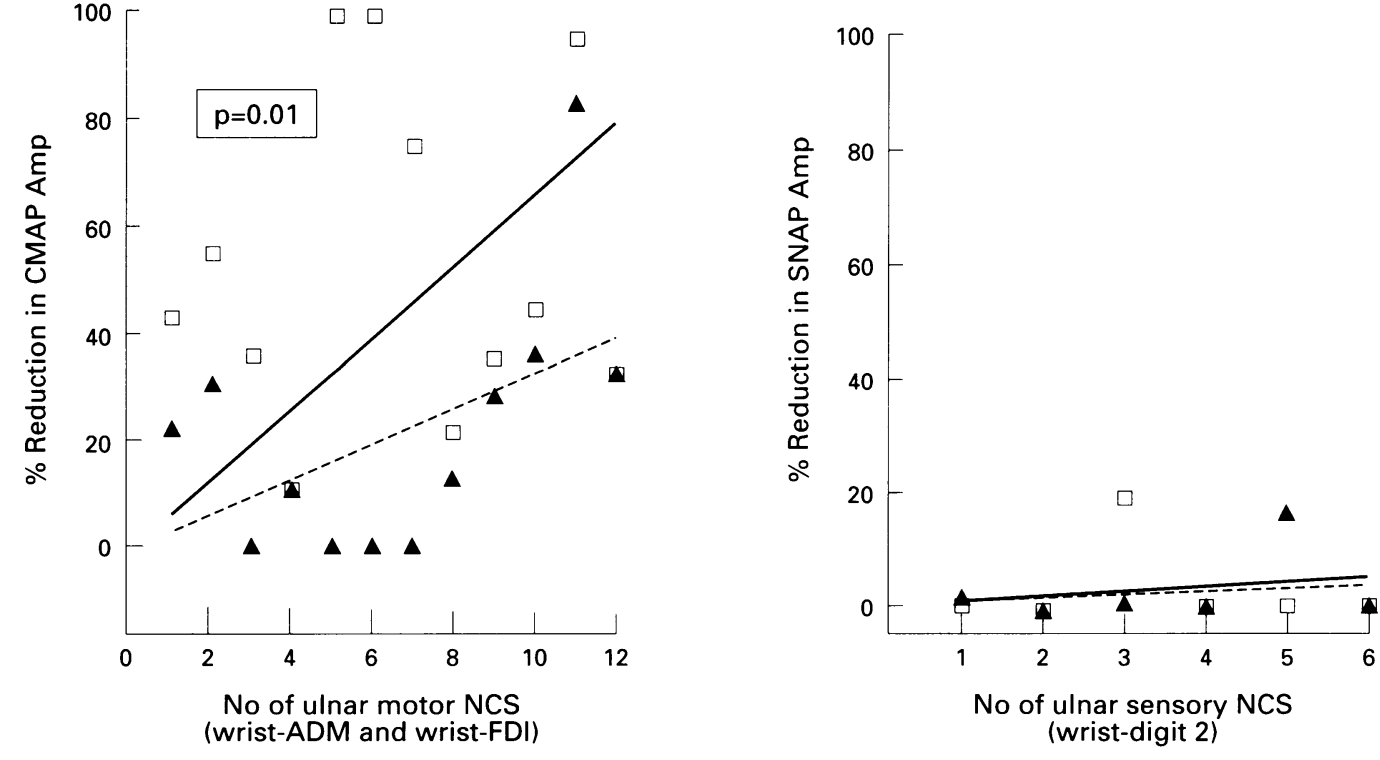

$\square-$ Side with entrapment

Side without entrapment 
Figure 7 Comparison of the CMAP amplitude in abductor digiti minimi $(A D M)$ and first dorsal interosseous (FDI) on the left $(L)$ side with those on the right $(R)$ side, in a patient with ulnar nerve entrapment only on the left side. Progressive reduction in CMAP amplitude occurred only on the left side (the side with entrapment), even though the starting amplitude was lower on the right side.

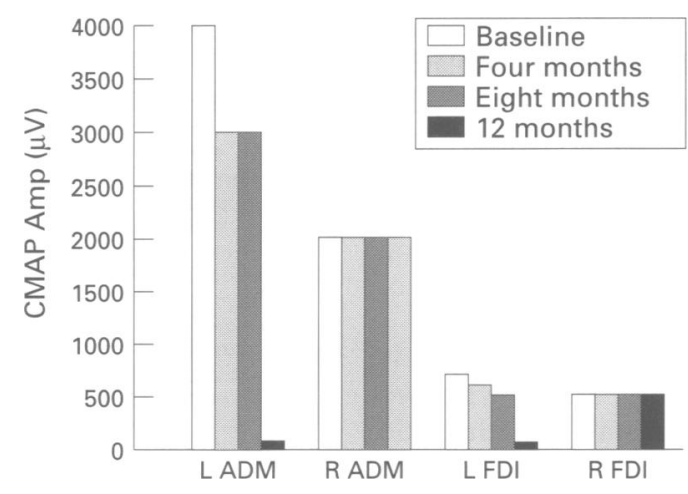

clinical studies of double crush had pitfalls in that they either lacked a rigorous definition of the two lesions or they failed to include a control population and to exclude the possibility that the findings were due to the chance occurrence of two common entities. ${ }^{347}$ Furthermore, cervical radiculopathies (usually C5-C6) may affect different fibre populations than entrapments (usually C8-T1) and should not cause sensory fibres to undergo axonal degeneration. Similarly, studies reporting the coexistence of thoracic outlet syndrome with a distal compressive neuropathy lacked objective evidence of the two lesions and failed to compare their patients with a control population. ${ }^{589}$ Although some experimental models of compressive neuropathy are purported to prove the double crush syndrome, none of these is an accurate model of clinical human entrapment neuropathy. ${ }^{10141518-20}$ The entity of double-crush syndrome, therefore, remains controversial. ${ }^{62122}$

Our prospective study had strict definitions for the two "lesions". All patients in this study were diagnosed as having amyotrophic lateral sclerosis based strictly on the criteria of the El Escorial World Federation of Neurology. ${ }^{23}$ More importantly, these patients were documented as having lower motor neuron lesions in the C8-T1 distribution, as evidenced by progressive loss of motor amplitudes in the ulnar innervated muscles of the hand. The adjacent travelling sensory fibres of the ulnar nerve remained normal over the period of the study, as shown by preserved distal SNAP amplitudes. The second lesion was strictly defined by a focal reduction of $\geqslant 10 \mathrm{~m} / \mathrm{s}$ in nerve conduction velocity across the elbow. No patient had any other definable lesion along the course of the nerve (such as distal ulnar neuropathy) except for ulnar nerve entrapment. All patients had sequential evaluations on both arms, thus serving as their own internal controls.

The elbow is often a site of minor trauma or traction to the ulnar nerve, so that "subclinical" ulnar nerve entrapment is not uncommon even in healthy subjects. This was evident from our study, in which $22 \%$ of the subjects had ulnar nerve entrapment by sensory nerve conduction studies. The sensory amplitudes remained unchanged over the period of the study, despite focal reductions in velocity across the elbow, confirming that the focal demyelinating change was not severe enough to cause axonal loss. Additionally, none of the patients had sensory or motor symptoms or signs limited to the ulnar nerve distribution; hence, a finding of ulnar nerve entrapment by nerve conduction studies was classified as subclinical.

The presence of amyotrophic lateral sclerosis produces an additional insult selectively to the motor component of ulnar nerve fibres. If the concept of double crush were correct, focal reductions in motor conduction velocities in the elbow segment should have occurred with progression of amyotrophic lateral sclerosis. However, motor nerves in motor neuron disease did not seem to be any more susceptible to entrapment at the elbow than were sensory nerves, thus casting doubt on the first part of the double crush hypothesis.

The second part of the hypothesis states that two sequential lesions may add to each other's effects. Although motor axonal loss was undoubtedly occurring over time in these patients with amyotrophic lateral sclerosis, ulnar nerve entrapment was not by itself producing significant axonal loss, as distal sensory amplitudes were preserved. And yet, motor axonal loss occurred more rapidly in patients with ulnar nerve entrapment than in those without entrapment. In the same patients, in whom a unilateral ulnar nerve entrapment was found, the side with entrapment had greater axonal loss than did the contralateral side without ulnar nerve entrapment. Figure 6 best illustrates this. Although the baseline CMAP amplitude was lower for the right side (suggesting greater motor axonal loss due to amyotrophic lateral sclerosis), after one year the amplitudes were lower on the left, presumably because there was additional entrapment. Thus one lesion by itself would not have produced that injury but the presence of two lesions together resulted in greater axonal loss.

The electrophysiological finding of ulnar nerve entrapment is frequent because the ulnar sensory and ulnar motor nerve fibres are exposed to external pressures at the elbow; in addition, because of their more superficial location, sensory fibres are more susceptible. However, segmental demyelination caused by ulnar nerve entrapment may not be of sufficient severity to result in distal axonal loss. An additional insult, a second lesion (the double crush), may be necessary to produce the axonal loss and perhaps make a subclinical entrapment into a clinical one.

Arguably, motor neuron disease with ulnar nerve entrapment does not represent a true "double crush", as the effect on nerve of motor neuron loss may not be the same as a proximal compressive lesion. However, as outlined in the initial report by Upton and $\mathrm{McComas},{ }^{2}$ any proximal nerve lesion that causes axonal dysfunction distally should produce the phenomenon of "double crush" when a second compressive lesion is added. Some of the experimental models of peripheral neuropathy associated with entrapment neuropathy have also called this paradigm "double crush". ${ }^{10141518-20}$ One advantage of our model is that in chronic degenerating diseases such as amyotrophic lateral sclerosis, the distal effects of a proximal "lesion" presumably occur over a long period of time; therefore, the affected motor fibres pre- 
sumably remain "diseased", rather than suffering an acute insult with complete Wallerian degeneration distally in seven to 10 days. This allows time to observe the effect of a second, compressive lesion. We cannot exclude an ulnar nerve entrapment presenting as entrapment in the motor fibres alone and causing axonal loss, given the greater susceptibility of sensory fibres due to their superficial location.

In conclusion, this electrophysiological study supports the notion that motor nerves with two insults undergo greater axonal loss than do nerves with one, but that the presence of one insult does not necessarily predispose a nerve to a second lesion.

1 Lishman WA, Russell WR. The brachial neuropathies. Lancet 1961;ii:941-6.

2 Upton ARM, McComas AJ. The double crush in nerveentrapment syndromes. Lancet 1973;ii:359-62.

3 Massey EW, Riley TL, Pleet AB. Coexistent carpal tunnel syndrome and cervical radiculopathy (double crush syndrome). South Med $\Im$ 1981;74:957-9.

4 Hurst LC, Weissberg D, Carroll RE. The relationship of the double crush to carpal tunnel syndrome (an analysis of 1,000 cases of carpal tunnel syndrome). $₹$ Hand Surg (Br) 1985;10-B:202-4.

5 Wood VE, Biondi J. Double-crush nerve compression in thoracic-outlet syndrome. F Bone foint Surg Am 1990; thoracic-o

6 Carroll RE, Hurst LC. The relationship of thoracic outlet syndrome and carpal tunnel syndrome. Clinical Orthopaedics and Related Research 1982;164:149-53.

7 Yu J, Brendler EM, Mentari A. Neurological disorders associated wtih carpal tunnel syndrome. Electromyogr Clin Neurophysiol 1979;19:27-32.

8 Lord JW, Rosati LM. Thoracic outlet syndrome. Ciba Clinical Symposia 1971;23:1.
9 Narkas AO. The role of thoracic outlet syndrome in double crush syndrome. Ann Hand Surg 1990;9:331-40.

10 Shimpo T, Gilliatt RW, Kennett RP, Allen PJ. Susceptibility to pressure neuropathy distal to a constricting ligature in the guinea-pig. $\boldsymbol{f}$ Neurol Neurosurg ing ligature in the guinea-pich

11 Mulder DW, Lambert EH, Bastron JA, Sprague RG. The neuropathies associated with diabetes mellitus: a clinical and electromyographic study of 103 unselected diabetic patients. Neurology 1961;11:275-84

12 Lambert EH, Mulder DW. Nerve conduction in GuillainBarré syndrome [abstract]. Electroencephalogr Clin Neurophysiol 1964;17:86.

13 Hopkins AP, Morgan-Hughes JA. The effect of local pressure in diphtheritic neuropathy. 7 Neurol Neurosurg Psychiatry 1969;32:614-23.

14 Nemoto K, Matsumoto N, Tazaki K-i, Horiuchi Y, Uchinishi K-i, Mori Y. An experimental study on the "double crush" hypothesis. $\mathcal{F}$ Hand Surg 1987;12A: 552-9.

15 Seiler WA, Schlegel R, MacKinnon SE, Dellon AL. Double crush syndrome: experimental model in the rat. Double crush syndrome: experim
Surgical Forum 1983;34:596-8.

16 MacKinnon SE. Double and multiple "crush" syndromes. Hand Clinics 1992;8:369-90.

17 Raynor EM, Shefner JM, Preston DC, Logigian EL. Sensory and mixed nerve conduction studies in the evaluation of ulnar neuropathy at the elbow. Muscle Nerve 1994;17:785-92.

18 MacKinnon SE, Dellon AL, Hudson AR, Hunter DA. Chronic nerve compression-an experimental model in the rat. Ann Plast Surg 1984;13:112-20.

19 O'Brien JP, MacKinnon SE, MacLean AR, Hudson AR, Dellon AL, Hunter DA. A model of chronic nerve compression in the rat. Ann Plast Surg 1987;19:430-5.

20 Reiners $\mathrm{K}$, Gilliatt RW, Harding AE, O'Neill JH. Regeneration following tibial nerve crush in the rabbit: the effect of proximal constriction. $\mathcal{f}$ Neurol Neurosurg the effect of proximal
Psychiatry 1987;50:6-11.

21 Murray-Leslie CF, Wright V. Carpal tunnel syndrome, humeral epicondylitis, and the cervical spine: a study of clinical and dimensional relations. BMF 1976;1:1439-42.

22 Swensen RS. The "double crush" syndrome. Neurology Chronicle 1994;4:1-6.

23 Criteria for diagnosis of amyotrophic lateral sclerosis. A workshop on "clinical limits of ALS" Escorial, Spain, 26-28 May, 1989. World Neurology 1990;5:12. 